\title{
Three Years of Medical Audit in a Psychiatric Unit
}

\author{
J. GuY Edwards, Consultant Psychiatrist, C. M. H. NunN, Consultant Psychiatrist, and B. S. RicketTs, \\ Consultant Psychiatrist, Royal South Hants Hospital, Southampton
}

For more than a decade psychiatrists have paid lip service to the idea that peer group medical audit might be valuable, but few have initiated regular audit meetings. This could be due partly to fear of criticism from colleagues and partly to a feeling that we can well do without additional, possibly time wasting, meetings. We in the Department of Psychiatry of the Royal South Hants Hospital have held monthly audit meetings for the last three years. We would like to report our experience of these, as we have found them to be supportive rather than threatening-despite our initial apprehension-and we believe that they have resulted in improvements in our service.

The initial impetus for developing a system of audit was provided by a prolonged crisis in our service between 1979 and 1982. In 1979 we took on sole responsibility for providing adult mental illness services for a catchment area population of over 300,000 , the psychogeriatric service being provided separately. Initially we were able to use only 60 beds. Recurrent financial crises greatly delayed the planned build-up of beds and staff, and as a result the Department was under great pressure. There was a greater than expected number of suicides among our in-patients which caused anxiety and despondency. Our consciences were slightly eased by an epidemiological study which showed that no increase in unnatural deaths had taken place in our catchment area, suggesting that some patients who planned to commit suicide did so in the unit rather than in the community.

We were nevertheless very concerned about the deaths and therefore consulted the Health Advisory Service. The HAS found "a high quality of care", but noted that the Department was "expected to take on too much without adequate resources". They also advised "a systematic procedure for the audit of untoward incidents (which) would be extremely useful to other psychiatric units elsewhere in the country". It was on this advice that our audit meetings were started.

At first meetings were held on an ad hoc basis whenever there was a successful suicide of an in-patient or of an outpatient known to our service - or whenever there was a 'near miss', for example when a young schizophrenic patient jumped out of a window and broke his legs. Meetings were open to consultants and senior registrars, and occasionally others involved in the care of the patient, such as senior nurses, were invited to attend. Confidence in the meetings grew and it became possible to express views more freely. Attendance was good with five to thirteen (30-87\%) of our colleagues turning up. The average attendance was nine. As a result of the build-up in confidence invitations to attend meetings have recently been extended to all junior medical staff in our Department. There has been debate about including colleagues from other disciplines, but the general feeling is that the attendance of others and increasing the size of the meeting might inhibit free discussion. Perhaps we are projecting our residual apprehensions about audit meetings.

When the crises in our unit subsided we decided to hold audit meetings on aspects of routine clinical care and at first selected cases at random from the in-patient and outpatient files. Whereas the consultant responsible for the patient presented the case to colleagues when discussing untoward incidents, the records of randomly selected cases are now handed to a consultant who does not know the patient and he is asked to review the management of the patient and then to present constructive criticisms to his colleagues in the presence of the responsible consultant. This arrangement also caused some initial apprehension, but in practice it has worked well and even untoward incidents are now dealt with in this way.

We found after a time that audit of randomly selected case records tended to produce comments centred around the adequacy of records, which although sometimes valuable could be seen as contributing little to the quality of patient care. We therefore progressed to a system in which each meeting is devoted to one aspect of our service. The aspects covered include day patient care, psychotherapy, rehabilitation, the hospital hostel for new long-stay patients, the psychiatric clinic in the University Health Centre, the management of alcoholics, the work of the Crisis Clinic, the treatment of patients requiring seclusion and others 'difficult to manage', and the assessment of cases with a diagnosis of 'no psychiatric abnormality'. We have also held meetings on the management of detained patients, prescription cards, a study of assaults occurring in the unit (which will be reported elsewhere) and variations in the length of stay of patients with similar diagnoses on different wards.

Many criticisms have emerged-some trivial, some important. These can be broadly categorised into those predominantly concerned with note keeping and those concerned with patient care (see Table). We believe that the former criticisms have promoted better record keeping while the latter have helped considerably in improving the overall standard of clinical practice. An important finding in one case for instance, was that there had been neglect in monitoring a patient's fluid balance which was ultimately attributable to under-staffing on the ward concerned. This has since been corrected. Perhaps the most important outcome of all has been that we have each been made aware that we are not alone in our failings and successes and that 
TABLE

Criticisms made at audit meetings: (128 cases)

\begin{tabular}{lc}
\hline & Number of criticisms \\
\hline Nature of criticism & \\
\hline & 27 \\
Predominantly concerned with record keeping & 18 \\
Inadequate notekeeping & 2 \\
Clerical omissions & \\
Unnecessary verbosity & 11 \\
Predominantly concerned with patient care & 8 \\
Inadequate follow-up and/or continuity of care & 5 \\
Inadequate social assessment & 4 \\
Inadequate liaison between staff & 4 \\
Inadequate physical investigations & 2 \\
Inadequate supervision of junior doctors & 2 \\
Inadequate drug treatment & 1 \\
Excessive delay in offering psychology appointment & 1 \\
Inappropriate diagnosis & 1 \\
Insufficient effort to find interpreter & 1 \\
Excessive physical investigation & 1 \\
Refusal of admission due to bed shortage & 1 \\
Excessive delay in recommending Section & 1 \\
Invalid consent to treatment & \\
Advice on drugs and driving not given & \\
\hline
\end{tabular}

medical colleagues can take a supportive and constructive view of both.

Minutes are taken at each audit meeting and are circulated to all those involved. In these minutes no patient is mentioned by name for reasons of confidentiality and no doctor or medical team is identified because of medico-legal concern. Wherever possible we reach overall conclusions and make recommendations for action. Such conclusions and recommendations are discussed at subsequent medical staff meetings and are brought to the attention of our Department of Psychiatry Management Team when necessary. Some of our recommendations fall by the wayside before they are translated into action. In such cases we can do little more than hope that the discussion leading to them has influenced the general climate of opinion. But in the majority of cases the meetings help us to formulate collective medical advice on the running of the service and provide a point of view that is not readily available from other sources. Our audit meetings are in effect a form of operational research. They would undoubtedly benefit from more sophisticated methodology, but in their present form they are flexible and responsive to immediate needs in a way that formal research cannot be.

Good case records do not necessarily mean high standards of patient care. They do, however, reflect the standard of care to some extent. Audit meetings of the kind we have described highlight problems that might not otherwise be identified and promote discussion that would not otherwise take place. Criticisms should be made in relation to the circumstances under which care is delivered. An incomplete psychiatric history of a patient seen in an emergency, for instance, should not be criticised, whereas more details would be expected on a patient who has been under prolonged care. What is wanted are the essentials of good care appropriate to the particular circumstances; no one expects the impossible.

Although criticisms in audit may be directed at individuals, it should not be forgotten that individual carers are no more than elements in highly complex systems. It is often elsewhere in the hierarchy that responsibility for deficiencies in the service lie. Consultants are legally responsible for failings in their junior colleagues, management and health authorities are responsible for an imbalance in staffing in different parts of a unit or in different specialities. In the ultimate analyis the Department of Health and Social Security and the Government are responsible for many grass root problems by imposing excessively heavy demands on National Health Service staff with inadequate resources to deal with them.

We can do little about problems that originate in the higher echelons of the NHS. We can, however, make significant changes in our everyday clinical practice. Our audit meetings at the Royal South Hants Hospital have increased our awareness of the many imperfections in our service and thereby reduced some of them. They have also provided each of us with a valued prop in an imperfect world. 